\title{
Re: Trends and practices for managing low-risk prostate cancer: A SEER-Medicare study
}

\author{
Peter C. Albertsen $\mathbb{1}^{1}$
}

Received: 10 May 2021 / Revised: 24 May 2021 / Accepted: 27 May 2021 / Published online: 9 June 2021

(c) The Author(s), under exclusive licence to Springer Nature Limited 2021

The management of localized prostate cancer has changed dramatically since prostate specific antigen (PSA) screening was first proposed in 1991 [1]. Prior to that clinicians assumed that most prostate cancers would eventually progress to metastatic disease although many older men often died with not of prostate cancer. Within 3 years of introducing PSA testing the incidence of this disease more than doubled in the US. Many of the men with newly diagnosed disease were urged to undergo either surgery or radiation to prevent cancer from metastasizing.

Hoffman et al. recently explored trends in the use of expectant management, active surveillance and active treatment in the management of men with newly diagnosed, localized prostate cancer [2]. They used the SEERMedicare database to identify all men age 66 and over who were diagnosed with low-risk prostate cancer. They captured prostate cancer treatments including surgical procedures, radiation, hormonal therapy and whether patients elected expectant management and/or active surveillance. The time period they studied was 2010-2013 and they found that expectant management had increased from 29 to $49 \%$.

Since 2013, several important studies have accelerated changes in prostate cancer management. Three major screening studies have shown us that PSA testing primarily identifies low volume, low grade disease [3]. The CAP trial revealed that three quarters of men in a screen naïve population will be diagnosed with Gleason Grade 1 disease [4]. Most of these cancers rarely progress to metastatic disease. Another $20 \%$ will be diagnosed with Gleason Grade Group 2 or 3 disease. Many of these cancers will growly slowly over a period of 15-20 years or longer. High grade prostate cancers are rare. Three

Peter C. Albertsen

albertsen@UCHC.edu

1 UConn Health, Farmington, CT, USA treatment trials have shown us that men with low volume, low grade cancers are not likely to benefit from either surgery or radiation because the vast majority of these men have indolent disease. As a consequence many clinicians now counsel patients about expectant management and active surveillance.

A careful review of Hoffman's article shows a number of specific findings. Men on expectant management tended to be under age 77. This makes sense because PSA screening declines as men age into their late 70's. PSA testing in men over age 77 is increasingly used to confirm suspected metastatic disease and not to screen for localized disease. Married men and men in higher socio-economic groups are more likely to follow current medical trends and inquire about expectant management. Finally, men with low PSA values and normal rectal exams are most likely to have indolent disease.

Hoffman et al. made another important observation. Within 3 years of diagnosis, $21.3 \%$ of the men in the expectant management cohort had switched to active treatment. They suggest that this decision is based upon evidence of disease progression, but they neglect to mention another important factor: patient (and likely physician) anxiety. Fear of cancer progression is a powerful motivator. Patients on active surveillance must be confident that their disease will not progress. Since 2013, we have accumulated much more data supporting this approach, but many patients still require a supportive spouse and physician to remain on an active surveillance protocol.

The future of prostate cancer screening remains uncertain. The historic paradigm of relying on an elevated PSA test has not worked. It has led to significant over diagnosis of indolent disease and has led the US Preventive Services Task Force to recommend against PSA screening [5]. Most European governments have failed to endorse PSA testing because of the lack of epidemiologic support. A recent review of US prostate cancer mortality statistics; however, suggests that PSA testing has been responsible for a $20 \%$ 
decline in prostate cancer mortality [6]. What treatments are driving this decline remains unclear.

To lower the rate of over diagnosis several changes to the PSA testing algorithm have been proposed including increasing the PSA threshold for biopsy and relying on more specific tests such as percent free PSA, the $4 \mathrm{~K}$ score or the PHI. In the UK, MRI has dramatically altered who gets biopsied. Our collective goal is to lower prostate cancer mortality. Identifying clinically significant disease early is the first critical step. If we rely on PSA testing alone we will fail to identify primarily men with high grade disease. These are the men who are at risk of disease progression and death from prostate cancer. These men require treatment; not expectant management. Expectant management is only a strategy to prevent harming men with indolent disease. Ideally, these men should never have been biopsied.

\section{Compliance with ethical standards}

Conflict of interest The authors declare no competing interest.
Publisher's note Springer Nature remains neutral with regard to jurisdictional claims in published maps and institutional affiliations.

\section{References}

1. Catalona WJ, Smith DS, Ratliff TL, Dodds KM, Coplen DE. Measurement of prostate-specific antigen in serum as a screening test for prostate cancer. N Eng J Med. 1991;324:1156-61.

2. Hoffman RM, Mott SL, McDowell BD, Anand ST, Nepple KG. Trends and practices for managing low risk prostate cancer: A SEER Medicare Study. Pros Ca and Pros Dis 2021; In press.

3. Albertsen PC. Prostate cancer screening and treatment: where have we come from and where are we going. BJUI. 2020;126:218-24.

4. Martin RM, Donovan JL, Turner EL, Metcalf C, Young GJ, Walsh EI, et al. Effect of low-intensity PSA-based screening intervention on prostate cancer mortality. JAMA. 2018;319:883-95.

5. Grossman DC, Curry SJ, Owens DK, Bibbins-Domingo K, Caughey AB, Davidson KW, et al. Screening for prostate cancer: US preventive services task force recommendation statement. JAMA. 2018;319:1901-13.

6. Welch HG, Albertsen PC. Reconsidering prostate cancer mortality - the future of PSA screening. N. Eng J Med. 2020;382: $1557-1563$. 